\title{
Comparison between Controlled Non-Adiabatic and $E \times B$ Concepts for Gyrotron Multistage Depressed Collectors
}

\author{
Chuanren Wu, Ioannis Gr. Pagonakis, Stefan Illy, Gerd Gantenbein, \\ Manfred Thumm, and John Jelonnek \\ Karlsruhe Institute of Technology, Karlsruhe, Germany, chuanren.wu@kit.edu
}

\begin{abstract}
Introduction
Gyrotrons are used as RF sources for ECH systems in nuclear fusion experiments. To obtain sufficient energy-gain factor, the future DEMOnstration fusion power plant demands highly efficient gyrotrons. The interaction between electron beam and microwave in a fusion gyrotron may have in the optimal case approximately $35 \%$ efficiency $\left(\eta_{\text {int }}\right)$. A large fraction of the input energy still remains in the spent electron beam. Up to $\eta_{\text {col }} \approx 60 \%$ of the spent beam energy can be recovered by a Single-stage Depressed Collector (SDC), which leads to an overall gyrotron efficiency of about $50 \%$ according to
\end{abstract}

$$
\eta_{\text {total }}=\frac{\eta_{\text {int }} \eta_{\mathrm{RF}}}{1-\left(1-\eta_{\text {int }}\right) \eta_{\text {col }}}
$$

where $\eta_{\mathrm{RF}} \approx 90 \%$ is assumed to be the ratio between the output and the generated RF power. To achieve $60 \%$ of gyrotron overall efficiency, the collector efficiency $\eta_{\text {col }}$ is required to be higher than $74 \%$. For this purpose, Multistage Depressed Collectors (MDC) should be introduced. In an MDC the spent electron beam will be sorted. Proper deceleration voltages (depression voltages) will be applied to each energy stage. Moreover, in order to reduce the thermal loading on the electrodes, the electron beam have to be eventually spread or swept on a large area.

Designing an MDC for fusion gyrotrons is nontrivial. Three major reasons are listed here. Firstly, fusion gyrotrons works under high magnetic field, which has a dependence on the cyclotron frequency as

$$
\omega_{\mathrm{c}}=\frac{e B_{0}}{\gamma m_{0}}, \text { with } \quad \gamma=\frac{1}{\sqrt{1-(v / c)^{2}}}
$$

For example, the Wendelstein 7-X $140 \mathrm{GHz}$ gyrotron requires more than $5 \mathrm{~T}$ field and the $170 \mathrm{GHz}$ ITER gyrotron requires almost $7 \mathrm{~T}$. At the collector entrance, the magnetic field from the gyrotron super-conducting coil is reduced, but still can be higher than $100 \mathrm{mT}$. The magnetic confinement in the collector prohibits the electron beam to be sorted or spread, since the prerequisites for the repulsion or the universal beam spread curve is not fulfilled. Secondly, electron beams in gyrotrons are annular. The beam thickness is small compared to the electron guiding center radii, while the magnetic flux enclosed in the hollow electron beam keeps invariant in a symmetric collector. As a consequence, during the unwinding of the magnetic field mentioned in the previous point, the expansion of the enclosed hollow region inside the beam is more eager than the increment of beam thickness itself. This would results in a large MDC geometry. Thirdly, due to the presence of the magnetic field, the trajectories of secondary electrons are harder to be controlled. The magnetic field may guide secondary as well as reflected electrons back to the interaction region.

Nevertheless, there are still two concepts for gyrotron MDCs. In the first one, the gyrotron magnetic field is unwound in a special way utilizing well-controlled non-adiabatic transitions [1], whereas in the second concept, the electrons are sorted by the plasma $\boldsymbol{E} \times \boldsymbol{B}$ drift [2]. In the present work, these two concepts will be discussed and compared with each other.

\section{Concept I: non-adiabatic demagnetization}

An intuitive solution to the issue with strong gyrotron magnetic field is to unwind it from $100 \mathrm{mT}$ until a low value, typically around $10 \mathrm{mT}$ [1]. If this process is adiabatic, a huge part of the electron transversal motion can be converted to the longitudinal one, however an adiabatic demagnetization will take place in a long distance, hence the collector geometry will be extremely long. Another side effect of an adiabatic magnetic field transition is that the electron beam is still bundled as before and weakly confined; mixed with the applied electric field, the electron trajectories are difficult to be manipulated. This observation is also in agreement with [3]. On the other hand, a non-adiabatic reduction of the magnetic field will shrink the transition and thus will reduce the length of the MDC significantly, for the price that the electron transversal velocity will not be completely converted, so that the optimal depression voltages may not be applied. As a trade-off, the unwinding of the magnetic field should be slightly non-adiabatic.

A side effect of the non-adiabatic transition is that it may spread the electron beam over the space by influencing the ratio of transversal / longitudinal velocities of each electron individually. Furthermore, a well-designed non-adiabatic transition may spatially modulate the electron trajectories according to their energy, which can be used as an energy sorting mechanism for MDC [4].

Since the system is axis symmetric, it can be modelled and simulated in 2D. Moreover, a so-called "effective potential" (strictly to say, the energy) $\varphi$ has been proposed in [1], which considers both electric and magnetic fields:

$$
\varphi=\frac{1}{2 m}\left(\frac{P_{\theta}-q r A_{\theta}}{r}\right)^{2}+q V,
$$

$P_{\theta}$ and $A_{\theta}$ are the azimuthal components of canonical momentum and magnetic vector potential, respectively; whereas $V$ is the electric potential. This is an elegant way to describe the system. However, to the knowledge of the authors, the $\varphi$ serves practically as a post-design diagnostic and explanation model rather than a design tool, since the synthesis of the field can still be tricky. 
As shown above, the first issue mentioned in the introduction can be solved through the non-adiabatic transition. In order to target the second issue, extra coils or ferromagnetic poles are inserted coaxially. The inserted magnets "pull" the electron beam to a smaller radius; while in addition, they can further reduce the off-axial magnetic field. There is no fundamental solutions to reduce the effects of secondary electrons except tuning the fields, looking for better materials or grooving the impacting area of electrodes. Typically $8 \%-10 \%$ of $\eta_{\text {col }}$ reduction by secondary electrons can be expected.

The maximal possible number of stages depends on how wide the electron beam can be spread in space. Broadly spreading the electron beam requires a weak magnetic field $(\ll 10 \mathrm{mT})$. In this case, the MDC may be sensitive to external magnetic fields and beam parameters such as beam current or misalignment. In the ideal case, a two-stage collector might have enough performance to boost the $\eta_{\text {total }}$ till $60 \%$ [4], while practically more stages might be necessary.

\section{Concept II: $E \times B$ drift}

A more promising MDC concept is the one using $E \times B$ drift. Its principle is shown in Fig. 1. The drift velocity is independent of particle mass, charge, energy and even the motion direction. In this model, each electron have an initial velocity component perpendicular to the direction of drift. The value of an electron's initial velocity depends on its initial energy. Since the same $\boldsymbol{E} \times \boldsymbol{B}$ drift is applied on each electron, the one with a higher initial velocity will travel further during the deceleration by the electric field until it lands on any electrode. The principle may allow arbitrary number of stages flexibly, as the electrons will always go to the desired electrodes, no matter how far the distances between stages are [5], which is in the previous concept not the case. This drift velocity also prevents secondary electrons to be reflected back to the interaction region, since most electrons are drifted back to the surface, where they were generated. In the worst case, a small fraction of electrons will be reflected only one stage back. This means that if there were more stages, the energy loss from those inter-stage secondary electrons would also be smaller.

There are several ways to obtain the $\boldsymbol{E} \times \boldsymbol{B}$ drift, it can be either an azimuthal $\boldsymbol{E}$ field with an axial $\boldsymbol{B}$ field $[2,6]$, or the contrary [7], or even some ideas out of the box that the annular beam can be transformed to one or more sheet beams $[5,8]$. Realistic simulations e.g. [6]

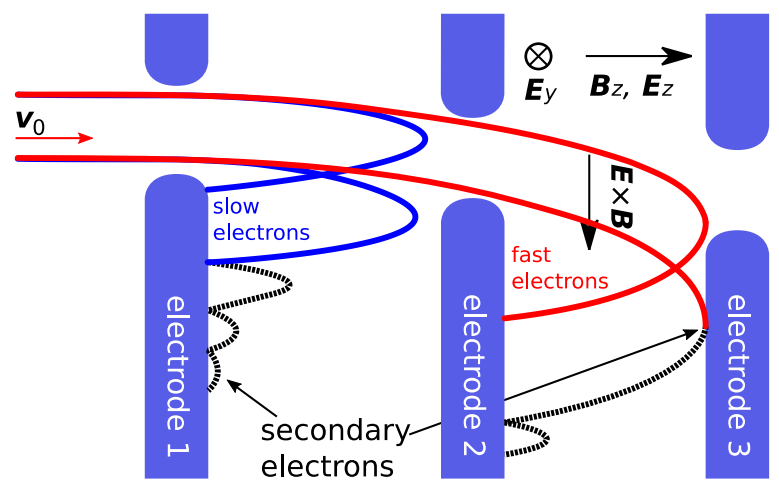

Fig. 1. Principle of the concept based on $\boldsymbol{E} \times \boldsymbol{B}$ drift

show that MDCs based on this concept can increase gyrotron efficiencies above $60 \%$ while the efficiency reduction by secondary electrons is very low.

\section{Conclusion}

The non-adiabatic demagnetization concept and the $E \times B$ drift concept for gyrotron MDC are compared. The comparison is concluded in the following table. For high-frequency gyrotrons the concept based on the $E \times B$ drift has more advantages and will be preferred.

\section{Acknowledgement}

This work has been carried out within the framework of the EUROfusion Consortium and has received funding from the Euratom research and training program 2014-2018 under grant agreement No. 633053. The views and opinions expressed herein do not necessarily reflect those of the European Commission.

\section{References}

1. Singh, A, Rajpatirana, S., Men, Y., Granatstein, V. L., Ives, R. L., Antolak, A. IEEE Trans. Plasma Sci., vol. 27, no. 2, pp. 490-502, 1999.

2. Pagonakis, I. G., Hogge, J. P., Alberti, S., Avramides, K. A., Vomvoridis, J. L. IEEE Trans. on Plasma Sci., vol. 36, no. 2, pp. 469-480, 2008

3. Read, M., Lawson, W., Dudas, A. J., Singh, A. IEEE Trans. Electron Devices, vol. 37, no. 6, pp. 1579-1589, 1990.

4. Wu, C., $\quad$ Pagonakis, I.Gr., Illy, S., Thumm, M., Gantenbein, G., Jelonnek, J. German Microwave Conf., pp. 365-368, 2016.

5. Pagonakis; I. Gr., Wu, C., Illy, S., Jelonnek, J. Phys. of Plasmas, 23, 043114, 2016

6. Wu, C., Pagonakis, I. Gr., Gantenbein, G., Illy, S., Thumm, M., Jelonnek, J. Int. Vacuum Electronics Conf. 2017

7. Louksha, O. I., Trofimov, P.A. International Vacuum Electronics Conf. 2017

8. Wu, C., Pagonakis, I. Gr., Gantenbein, G., Illy, S., Thumm, M., Jelonnek, J. Phys. of Plasmas 24, 043102, 2017

\begin{tabular}{|c|c|c|}
\hline $\begin{array}{c}\text { Magnetic } \\
\text { Field }\end{array}$ & $\begin{array}{c}\text { Non-Adiabatic Concept } \\
\text { Non-adiabatic unwound to weak magnetic } \\
\text { field typically } \leq 10 \mathrm{mT}\end{array}$ & $\begin{array}{c}\text { Gently unwound to moderate magnetic field } \\
\text { typically 30-60 mT for fusion gyrotrons }\end{array}$ \\
\hline More Stages & Hard & Straightforward \\
\hline $\begin{array}{c}\text { Secondary } \\
\text { Electrons }\end{array}$ & $\begin{array}{c}\text { Not easy to handle } \\
\text { May reduce } \eta \text { a lot (typically } 8-10 \%)\end{array}$ & $\begin{array}{c}\text { The same drift is applied also on secondary electrons } \\
\text { Small influence on efficiency (1 \% if optimized [6]) }\end{array}$ \\
\hline Tolerances & $\begin{array}{c}\text { Might be sensitive to electron beam } \\
\text { parameters and external magnetic fields }\end{array}$ & $\begin{array}{c}\text { May tolerate weak external magnetic fields and } \\
\text { slightly misaligned beam due to the moderate } \boldsymbol{B} \text { field }\end{array}$ \\
\hline $\begin{array}{c}\text { Design } \\
\text { Approaches }\end{array}$ & $\begin{array}{c}\text { Rotationally symmetric (2D description) } \\
\text { Effective potential aided } \\
\text { design and optimization }\end{array}$ & $\begin{array}{c}\text { Not axisymmetric (3D description) } \\
\text { Azimuthal } \boldsymbol{E} \text { field [2,6] or } \boldsymbol{B} \text { field }[7] \\
\text { Transformation to sheet beam [5,8] }\end{array}$ \\
\hline
\end{tabular}

